\title{
Polariton interactions in microcavities with atomically thin semiconductor layers
}

\author{
Olivier Bleu $\odot$, Guangyao Li $\odot$, Jesper Levinsen $\odot$, and Meera M. Parish $\odot$ \\ School of Physics and Astronomy, Monash University, Victoria 3800, Australia \\ and ARC Centre of Excellence in Future Low-Energy Electronics Technologies, Monash University, Victoria 3800, Australia
}

(Received 3 April 2020; revised 30 June 2020; accepted 6 October 2020; published 4 November 2020)

\begin{abstract}
We investigate the interactions between exciton-polaritons in $N$ two-dimensional semiconductor layers embedded in a planar microcavity. In the limit of low-energy scattering, where we can ignore the composite nature of the excitons, we obtain exact analytical expressions for the spin-triplet and spin-singlet interaction strengths, which go beyond the Born approximation employed in previous calculations. Crucially, we find that the strong light-matter coupling enhances the strength of polariton-polariton interactions compared to that of the exciton-exciton interactions, due to the Rabi coupling and the small photon-exciton mass ratio. We furthermore obtain the dependence of the polariton interactions on the number of layers $N$, and we highlight the important role played by the optically dark states that exist in multiple layers. In particular, we predict that the singlet interaction strength is stronger than the triplet one for a wide range of parameters in most of the currently used transition metal dichalcogenides. This has consequences for the pursuit of polariton condensation and other interaction-driven phenomena in these materials.
\end{abstract}

DOI: 10.1103/PhysRevResearch.2.043185

\section{INTRODUCTION}

Microcavity exciton-polaritons (polaritons) are neutral quasiparticles that arise from the strong coupling between semiconductor excitons (bound electron-hole pairs) and cavity photon resonances. Due to their excitonic component, polaritons interact with each other, in contrast to bare photons in vacuum. This interaction is the cornerstone of a variety of observed phenomena ranging from optical parametric scattering [1] and bistability [2], to Bose-Einstein condensation [3,4], superfluidity [5], and the formation of quantized vortices [6]. Hence, semiconductor microcavities are fruitful platforms to investigate two-dimensional (2D) quantum fluids of light [7-10].

Atomically thin semiconductors in the form of transition metal dichalogenides (TMDs) have recently emerged as promising materials for realizing polaritonic phenomena at room temperature, due to the large exciton binding energies in TMD monolayers [11-14]. Furthermore, TMDs can be made nearly disorder free, unlike organic materials [15], and they can be externally tuned using electrostatic gating [14], which is an essential tool for any future optoelectronic devices [16]. Already, a strong exciton-photon (Rabi) coupling has been observed in both TMD monolayer [17-20] and multilayer structures $[21,22]$. In particular, the use of multilayer van der Waals heterostructures can generate large Rabi couplings [23] as well as provide routes towards engineering other material

Published by the American Physical Society under the terms of the Creative Commons Attribution 4.0 International license. Further distribution of this work must maintain attribution to the author(s) and the published article's title, journal citation, and DOI. properties [24]. However, it is an open and nontrivial question how the polariton-polariton interactions depend on experimental parameters such as the light polarization [25] and the number of layers in these systems. The answer to this question impacts the highly active investigation of interaction-induced nonlinear optical properties [26-30] and the ongoing quest [31] to realize polariton condensation in pure TMD systems.

In this paper we address this question by studying the effective interactions between polaritons in a system of $N$ identical 2D layers embedded in a planar microcavity. A key simplification of our work is to assume that the energy scale of polariton-polariton scattering is smaller than the exciton binding energy $\varepsilon_{B}^{X}$, thus allowing us to treat the excitons as bosons with contact interactions $[32,33]$. This is a reasonable assumption in the case of TMD layers, where the exciton binding energy is much larger than all other relevant energy scales $[14,23]$. Solving the scattering problem of two lower polaritons in the limit of zero momentum, we obtain the following exact expression for the polariton-polariton interaction strength:

$$
T_{\sigma \sigma^{\prime}}=\frac{4 \pi \hbar^{2} X_{0}^{4}}{m_{X} N \ln \left(\frac{\varepsilon_{\sigma \sigma^{\prime}}}{2\left|E_{0}^{L}\right|}\right)} \equiv \begin{cases}\alpha_{1}, & \sigma=\sigma^{\prime}, \\ \alpha_{2}, & \sigma \neq \sigma^{\prime} .\end{cases}
$$

Here $\varepsilon_{\sigma \sigma^{\prime}}>0$ are the energies associated with the spin-triplet $\left(\sigma=\sigma^{\prime}\right)$ and spin-singlet $\left(\sigma \neq \sigma^{\prime}\right)$ exciton scattering lengths, where $\sigma= \pm$ encodes the pseudospin (circular polarization) of the exciton (photon). $m_{X}$ is the exciton mass, while $X_{0}^{2}$ and $E_{0}^{L}$ are, respectively, the exciton fraction and the energy (relative to the exciton energy) of the zero-momentum lower polariton, which depend on the number of layers $N$ via the exciton-photon Rabi coupling.

Crucially, Eq. (1) differs from the case of exciton interactions where the scattering has been shown to decrease 
logarithmically with collision energy $[32,33]$, as expected for 2D quantum particles with short-range interactions $[34,35]$. Hence the strong light-matter coupling enhances the polariton-polariton interaction strength with respect to the corresponding exciton-exciton interaction strength, which is a major qualitative difference from previous treatments based on the Born approximation [9,36,37]. Indeed, within the Born approximation, the coupling to light decreases the interaction strength due to the reduced exciton fraction, a feature which has become a central tenet of polariton physics [9]. Our result shows that, generically, the converse is true. Moreover, this simple analytic expression only depends on measurable parameters and can be universally applied to a range of TMD materials and even single semiconductor quantum wells where $\left|E_{0}^{L}\right| \ll \varepsilon_{B}^{X}$. Thus, Eq. (1) is a key result of this work.

The paper is organized as follows. The model is introduced in Sec. II where we highlight the nontrivial subtleties of multilayer systems. In Sec. III we present the derivation of the polariton-polariton scattering $T$ matrix, and explain how it differs from the standard low energy quantum scattering in 2D. Finally, we apply our results to different TMD materials and discuss the implications for experiments in Sec. IV. A brief summary and our conclusions are given in Sec. V. Additional information and technical details are provided in Appendices A, B, and C.

\section{MODEL}

\section{A. Dark, bright, and polariton states}

We start with the single-polariton Hamiltonian that describes the coupling between the cavity photon and $N$ monolayer excitonic modes:

$$
\begin{aligned}
\hat{H}_{0}= & \sum_{\mathbf{k}, \sigma} E_{\mathbf{k}}^{C} \hat{c}_{\mathbf{k} \sigma}^{\dagger} \hat{c}_{\mathbf{k} \sigma}+\sum_{\mathbf{k}, \sigma} \sum_{n=1}^{N} E_{\mathbf{k}}^{X} \hat{x}_{\mathbf{k} \sigma, n}^{\dagger} \hat{x}_{\mathbf{k} \sigma, n} \\
& +\frac{\hbar g_{R}}{2} \sum_{\mathbf{k}, \sigma} \sum_{n=1}^{N}\left(\hat{x}_{\mathbf{k} \sigma, n}^{\dagger} \hat{c}_{\mathbf{k} \sigma}+\hat{c}_{\mathbf{k} \sigma}^{\dagger} \hat{x}_{\mathbf{k} \sigma, n}\right),
\end{aligned}
$$

where $\hat{c}_{\mathbf{k} \sigma}\left(\hat{c}_{\mathbf{k} \sigma}^{\dagger}\right)$ and $\hat{x}_{\mathbf{k} \sigma, n}\left(\hat{x}_{\mathbf{k} \sigma, n}^{\dagger}\right)$ are bosonic annihilation (creation) operators of cavity photons and monolayer excitons, respectively, with in-plane momentum $\hbar \mathbf{k}$ and layer index $n$. The kinetic energies at low momenta are $E_{\mathbf{k}}^{C}=\hbar^{2} k^{2} / 2 m_{C}+\delta$ and $E_{\mathbf{k}}^{X}=\hbar^{2} k^{2} / 2 m_{X}$, where $k \equiv|\mathbf{k}|$ and we measure energies with respect to the exciton energy at zero momentum. Thus, $\delta$ is the photon-exciton detuning, while $m_{C}$ is the photon mass. Here, for simplicity, we consider identical monolayers that are located at the maxima of the electric field within the cavity, so that both $E_{\mathrm{k}}^{X}$ and the exciton-photon coupling $g_{R}$ are independent of $n$. However, it is straightforward to generalize our results to the case of a layer-dependent light-matter coupling. We furthermore assume that $g_{R}$ is independent of polarization/spin and we neglect any splittings between the longitudinal and transverse modes of the excitons [38-40] and photons [41]. Hence, there is no spin-orbit coupling [42-45] in our model, but such a single-particle effect should not strongly affect the short-distance two-body scattering processes considered here.
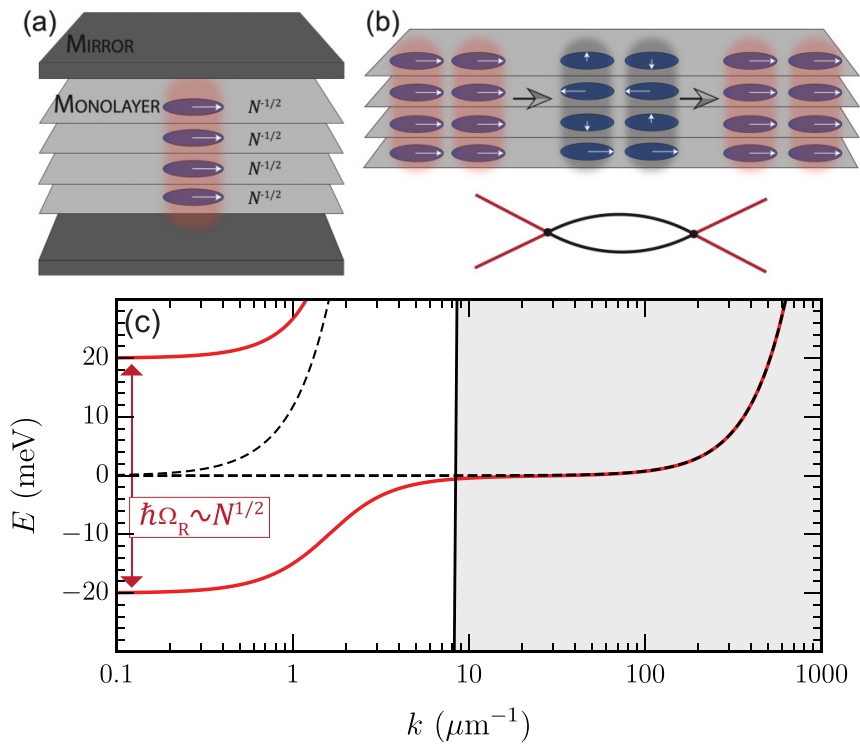

FIG. 1. (a) Schematic illustration of the microcavity structure with $N$ embedded monolayers, where $N=4$. A polariton consists of a cavity photon (shaded red) and a superposition of $N$ in-phase $2 \mathrm{D}$ excitons (blue ellipses), where the relative phase of each exciton is represented by an in-plane arrow. (b) Example of a scattering process involving intermediate dark states which are uncoupled to light. (c) Polariton dispersion (red) at zero detuning, together with the uncoupled cavity photon and exciton modes (dashed black lines). For high momenta $k>\epsilon_{X} / \hbar c$ (shaded region), where $\epsilon_{X}$ is the exciton energy and $c$ is the speed of light, the exciton is far detuned in energy from the photon and thus essentially uncoupled. We use the parameters for $\mathrm{MoSe}_{2}$ (see Table I) with $\epsilon_{X}=1.66 \mathrm{eV}$ [21] and $N=4$ layers.

The spin-degenerate eigenstates of Eq. (2) consist of two polariton modes (upper and lower branches) and $N-1$ dark states which are decoupled from light $[46,47]$. The multilayer system is frequently described by a two-coupled-mode exciton-photon model with a renormalized Rabi coupling [9], but here we keep track of the complete structure of the eigenstates which is of crucial importance when we consider polariton interactions below. Since only the bright states [inphase superpositions of all monolayer excitons, as depicted in Fig. 1(a)] couple to light, one can rewrite the Hamiltonian in the corresponding convenient basis:

$$
\begin{aligned}
\hat{H}_{0}= & \sum_{\mathbf{k}, \sigma}\left[E_{\mathbf{k}}^{C} \hat{c}_{\mathbf{k} \sigma}^{\dagger} \hat{c}_{\mathbf{k} \sigma}+E_{\mathbf{k}}^{X}\left(\hat{b}_{\mathbf{k} \sigma}^{\dagger} \hat{b}_{\mathbf{k} \sigma}+\sum_{l=1}^{N-1} \hat{d}_{\mathbf{k} \sigma, l}^{\dagger} \hat{d}_{\mathbf{k} \sigma, l}\right)\right] \\
& +\frac{\hbar \Omega_{R}}{2} \sum_{\mathbf{k}, \sigma}\left(\hat{b}_{\mathbf{k} \sigma}^{\dagger} \hat{c}_{\mathbf{k} \sigma}+\hat{c}_{\mathbf{k} \sigma}^{\dagger} \hat{b}_{\mathbf{k} \sigma}\right),
\end{aligned}
$$

where $\hat{b}_{\mathbf{k} \sigma}$ and $\hat{d}_{\mathbf{k} \sigma, l}$ are the annihilation operators for bright and dark states, respectively, which are related to the bare monolayer exciton operators via the unitary transformation:

$$
\hat{d}_{\mathbf{k} \sigma, l}=\sum_{n=1}^{N} u_{l n} \hat{x}_{\mathbf{k} \sigma, n}, \quad \hat{b}_{\mathbf{k} \sigma} \equiv \hat{d}_{\mathbf{k} \sigma, N}=\sum_{n=1}^{N} \frac{\hat{x}_{\mathbf{k} \sigma, n}}{\sqrt{N}},
$$


with $u_{l n}=\frac{1}{\sqrt{N}} e^{i 2 \pi n l / N}$ [48]. The multilayer nature of the bright states gives rise to an enhanced Rabi coupling $\hbar \Omega_{R}=$ $\hbar g_{R} \sqrt{N}$, thus making it easier to access the strong-coupling regime in a multilayer structure. We emphasize that the present dark states consist of superpositions of monolayer bright excitons. Thus, they should not be confused with spinforbidden dark excitons which can exist in free monolayers and have a different spectral energy [49,50].

The decomposition of the Hamiltonian into the basis of dark and bright excitons in Eq. (3) allows us to arrive at the diagonal form of the exciton-photon Hamiltonian:

$$
\hat{H}_{0}=\sum_{\mathbf{k}, \sigma}\left[E_{\mathbf{k}}^{L} \hat{L}_{\mathbf{k} \sigma}^{\dagger} \hat{L}_{\mathbf{k} \sigma}+E_{\mathbf{k}}^{U} \hat{U}_{\mathbf{k} \sigma}^{\dagger} \hat{U}_{\mathbf{k} \sigma}+\sum_{l=1}^{N-1} E_{\mathbf{k}}^{X} \hat{d}_{\mathbf{k} \sigma, l}^{\dagger} \hat{d}_{\mathbf{k} \sigma, l}\right],
$$

with $\hat{L}(\hat{U})$ the lower (upper) polariton annihilation operators defined in the standard way:

$$
\left(\begin{array}{l}
\hat{L}_{\mathbf{k} \sigma} \\
\hat{U}_{\mathbf{k} \sigma}
\end{array}\right)=\left(\begin{array}{cc}
X_{\mathbf{k}} & C_{\mathbf{k}} \\
-C_{\mathbf{k}} & X_{\mathbf{k}}
\end{array}\right)\left(\begin{array}{l}
\hat{b}_{\mathbf{k} \sigma} \\
\hat{c}_{\mathbf{k} \sigma}
\end{array}\right)
$$

Here $E_{\mathbf{k}}^{U, L}$ are the polariton eigenenergies [see Fig. 1(c)],

$$
E_{\mathbf{k}}^{U, L}=\frac{1}{2}\left(E_{\mathbf{k}}^{X}+E_{\mathbf{k}}^{C} \pm \sqrt{\left(E_{\mathbf{k}}^{C}-E_{\mathbf{k}}^{X}\right)^{2}+\hbar^{2} \Omega_{R}^{2}}\right),
$$

and $X_{\mathbf{k}}, C_{\mathbf{k}}$ are the Hopfield coefficients, corresponding to exciton and photon fractions:

$$
X_{\mathbf{k}}^{2}=\frac{1}{2}\left(1+\frac{E_{\mathbf{k}}^{C}-E_{\mathbf{k}}^{X}}{E_{\mathbf{k}}^{U}-E_{\mathbf{k}}^{L}}\right), \quad C_{\mathbf{k}}^{2}=1-X_{\mathbf{k}}^{2} .
$$

\section{B. Exciton-exciton interactions}

Since the layer spacing is typically larger than the exciton size, we may assume that the interactions between excitons only occur within the same layer. Furthermore, if the scattering energy is small compared to the exciton binding energy (as is the case in TMDs [14]), then we can describe the exciton interactions with an $s$-wave contact potential,

$$
\hat{V}=\sum_{n=1}^{N} \sum_{\substack{\mathbf{k}, \mathbf{k}^{\prime}, \mathbf{q} \\ \sigma, \sigma^{\prime}}} \frac{g_{\sigma \sigma^{\prime}}}{2} \hat{x}_{\mathbf{k}+\mathbf{q} \sigma, n}^{\dagger} \hat{x}_{\mathbf{k}^{\prime}-\mathbf{q} \sigma^{\prime}, n}^{\dagger} \hat{x}_{\mathbf{k}^{\prime} \sigma^{\prime}, n} \hat{x}_{\mathbf{k} \sigma, n},
$$

since at large separation the excitons have van der Waals interactions, which are short range [51]. The "bare" spindependent coupling strength $g_{\sigma \sigma^{\prime}}$ is independent of layer index $n$ since we have assumed that the monolayers are identical. Also, we have set the monolayer area to 1. Our approach is different from determining the exciton-exciton interaction strength within the Born approximation, as in previous works $[36,37,52-56]$. That approximation effectively estimates $g_{\sigma \sigma^{\prime}}$ from the microscopic structure of the excitons, whereas here we solve the low-energy scattering problem exactly and treat $g_{\sigma \sigma^{\prime}}$ as a bare parameter that must be related to experimental observables [57]. As such, we impose a cutoff $\Lambda$ on the relative scattering momentum, which we will send to infinity at the end of the calculation.
Transforming to the bright-dark exciton basis using Eq. (4), the interaction term becomes

$$
\hat{V}=\sum_{\left\{l_{j}\right\}} \delta_{\mathcal{M}} \sum_{\substack{\mathbf{k}, \mathbf{k}^{\prime}, \mathbf{q} \\ \sigma, \sigma^{\prime}}} \frac{g_{\sigma \sigma^{\prime}}}{2 N} \hat{d}_{\mathbf{k}+\mathbf{q} \sigma, l_{1}}^{\dagger} \hat{d}_{\mathbf{k}^{\prime}-\mathbf{q} \sigma^{\prime}, l_{2}}^{\dagger} \hat{d}_{\mathbf{k}^{\prime} \sigma^{\prime}, l_{3}} \hat{d}_{\mathbf{k} \sigma, l_{4}}
$$

where $\left\{l_{j}\right\}=\left\{l_{1}, l_{2}, l_{3}, l_{4}\right\}$. The Kronecker delta $\left(\delta_{\mathcal{M}}=1\right.$ if $\mathcal{M}=0, \delta_{\mathcal{M}}=0$ otherwise) encodes the phase selection rule for binary scatterings illustrated in Fig. 1(b), where $\mathcal{M}=$ $\operatorname{Mod}\left[l_{1}+l_{2}-l_{3}-l_{4}, N\right]$. It is worth noting that the interaction coupling constant is reduced by the factor of $1 / N$ in the new bright-dark-states basis. Moreover, written in this form, Eq. (10) can involve a huge number of terms $\left(N^{3}\right.$ for each spin channel). This highlights the complexity of the scattering processes which can occur in any multilayer structure in the strong-coupling regime.

\section{POLARITON-POLARITON SCATTERING}

To investigate polariton-polariton interactions, we consider the two-body scattering problem at zero center-ofmass momentum. The two-particle states are $\left|A_{\sigma}, B_{\sigma^{\prime}}, \mathbf{k}\right\rangle=$ $\hat{A}_{-\mathbf{k} \sigma}^{\dagger} \hat{B}_{\mathbf{k} \sigma^{\prime}}^{\dagger}|0\rangle$, where the operators $\hat{A}, \hat{B}$ can correspond to lower polaritons $\hat{L}$, upper polaritons $\hat{U}$, or dark-exciton operators $\hat{d}_{l}$, with $l=1,2, \ldots, N-1$. To proceed, we employ the $T$-matrix operator, which is given by the Born series

$$
\hat{T}(E)=\hat{V}+\hat{V} \frac{1}{E-\hat{H}_{0}+i 0} \hat{V}+\cdots,
$$

where $E$ is the scattering energy and $+i 0$ represents an infinitesimal positive imaginary part. The interaction strength for lower polaritons is then given by the matrix element

$$
T_{\sigma \sigma^{\prime}}(k) \equiv \frac{\left\langle L_{\sigma}, L_{\sigma^{\prime}}, \mathbf{k}^{\prime}\left|\hat{T}\left(2 E_{\mathbf{k}}^{L}\right)\right| L_{\sigma}, L_{\sigma^{\prime}}, \mathbf{k}\right\rangle}{1+\delta_{\sigma \sigma^{\prime}}},
$$

with the on-shell condition $\left|\mathbf{k}^{\prime}\right|=|\mathbf{k}|=k$. Here the normalization factor in the denominator accounts for scattering between identical particles. The Born approximation to the interaction strength corresponds to keeping only the first term in the series, which gives $g_{\sigma \sigma^{\prime}} X_{\mathbf{k}}^{4} / N$. However, higher order terms will significantly modify this result since they can involve scattering into dark intermediate states, as illustrated in Fig. 1(b).

Remarkably, we find that the low-energy polariton $T$ matrix takes the simple form (see Appendix A for the detailed

TABLE I. Experimental values used in Figs. 2 and 3 for the monolayer Rabi splitting $\left(\hbar g_{R}\right)$, exciton $\left(\varepsilon_{B}^{X}\right)$, and biexciton $\left(\varepsilon_{B}^{X X}\right)$ binding energies, all in units of $\mathrm{meV}$.

\begin{tabular}{lcccc}
\hline \hline Material & $\mathrm{MoSe}_{2}$ & $\mathrm{WSe}_{2}$ & $\mathrm{MoS}_{2}$ & $\mathrm{WS}_{2}$ \\
\hline$\hbar g_{R}$ & $20[21]$ & $23.5[19]$ & $46[17]$ & $70[18]$ \\
$\varepsilon_{B}^{X}$ & $470[21]$ & $370[11,59]$ & $960[17]$ & $700[18]$ \\
$\varepsilon_{B}^{X X}$ & $20[60]$ & $52[59]$ & $70[61]$ & $53[62]$ \\
\hline \hline
\end{tabular}



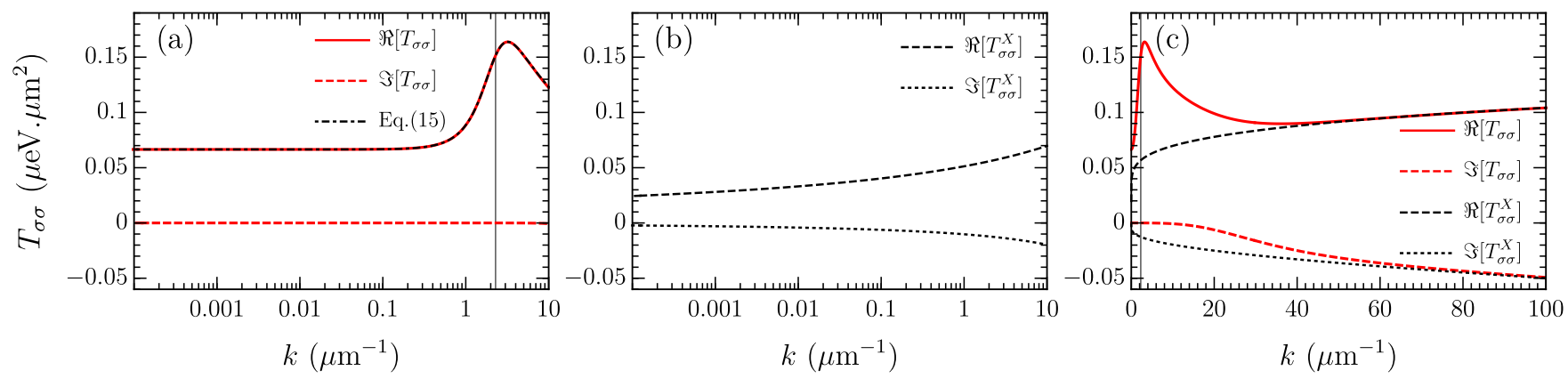

FIG. 2. Polariton and exciton triplet elastic scattering as a function of relative momentum. (a) Real and imaginary parts of the full polariton $T$ matrix, Eq. (13), together with the analytical formula in the small photon mass approximation, Eq. (16). (b) Low-energy exciton triplet $T$ matrix $T_{\sigma \sigma}^{X}(k)$, Eq. (17). (c) Polariton and exciton $T$ matrices on a larger momentum scale. We use the MoSe $\mathrm{P}_{2}$ parameters with $m_{X}=1.14 m_{0}$ [58], $m_{C}=10^{-5} m_{0}$ [28], $\delta=0$, and $N=1$, giving the inflection wave vector $q_{0} \simeq 2.29 \mu \mathrm{m}^{-1}$ [thin vertical line in (a) and (c)].

calculation)

$$
T_{\sigma \sigma^{\prime}}(k)=\frac{X_{\mathbf{k}}^{4}}{\frac{N}{g_{\sigma \sigma^{\prime}}}-\Pi\left(2 E_{\mathbf{k}}^{L}\right)} .
$$

Here the one-loop polarization bubble $\Pi(E)$ is extremely well approximated by that of $N$ exciton pairs:

$$
\Pi(E) \simeq N \sum_{\mathbf{q}}^{\Lambda} \frac{1}{E-2 E_{\mathbf{q}}^{X}+i 0},
$$

since the exciton scattering is dominated by large momenta where the photon is far off resonant [see Fig. 1(c)]. This is a consequence of the small photon-exciton mass ratio $m_{C} / m_{X}$ $\simeq 10^{-5}$ (see Appendix B).

To obtain cutoff-independent results, we relate the bare couplings to physical observables as follows [35]:

$$
\frac{1}{g_{\sigma \sigma^{\prime}}}=-\sum_{\mathbf{q}}^{\Lambda} \frac{1}{\varepsilon_{\sigma \sigma^{\prime}}+2 E_{\mathbf{q}}^{X}},
$$

where we have introduced the physical energy scales $\varepsilon_{\sigma \sigma^{\prime}}=$ $\frac{\hbar^{2}}{2 m_{r} a_{\sigma \sigma^{\prime}}^{2}}$ related to the $2 \mathrm{D}$ exciton $s$-wave scattering lengths $a_{\sigma \sigma^{\prime}}$ and the two-exciton reduced mass $m_{r}=m_{X} / 2$. Note that the scattering parameters are intrinsic to the monolayer and are independent of $N$. In the singlet case, $\varepsilon_{+-}=\varepsilon_{-+}=\varepsilon_{B}^{X X}$ corresponds to the binding energy of the biexciton (bound state of two excitons). Due to Pauli exclusion, there is no triplet biexciton state, but the triplet scattering length is well defined and is of the order of the $2 \mathrm{D}$ exciton Bohr radius $a_{\sigma \sigma} \sim a_{B}$ (see Appendix C); hence we have $\varepsilon_{\sigma \sigma} \sim \varepsilon_{B}^{X}$.

Inserting Eq. (15) into Eq. (13) and taking the limit $\Lambda \rightarrow$ $\infty$, one obtains the cutoff-independent $T$ matrix

$$
T_{\sigma \sigma^{\prime}}(k)=\frac{4 \pi \hbar^{2} X_{\mathbf{k}}^{4}}{m_{X} N \ln \left(\frac{\varepsilon_{\sigma \sigma^{\prime}}}{2\left|E_{\mathbf{k}}^{L}\right|}\right)} .
$$

The limit $k \rightarrow 0$ finally yields $T_{\sigma \sigma^{\prime}}$ in Eq. (1), which gives the lower polariton effective interaction "constants" for the triplet $\left(\alpha_{1}\right)$ and singlet $\left(\alpha_{2}\right)$ channels.

\section{A. Comparison between exciton-exciton and polariton-polariton scattering}

Equations (1) and (16) are key results of this work, since they imply that the low-momentum polariton interaction strength is enhanced compared to that of monolayer excitons, which is in sharp contrast to the behavior predicted by the Born approximation $[9,36,37]$. To see this, note that in the absence of light-matter coupling, Eq. (16) reduces to the usual 2D two-body $T$ matrix for quantum particles in a monolayer [34],

$$
T_{\sigma \sigma^{\prime}}^{X}(k)=\frac{4 \pi \hbar^{2}}{m_{X}} \frac{1}{\ln \left(\frac{\varepsilon_{\sigma \sigma^{\prime}}}{2 E_{\mathbf{k}}^{X}}\right)+i \pi},
$$

which describes the interactions of excitons. In Fig. 2 we compare the momentum dependence of the low-energy triplet polariton and exciton $T$ matrices for parameters corresponding to a $\mathrm{MoSe}_{2}$ monolayer (see Table I). Crucially, we see that the strength of polariton-polariton elastic scattering $T_{\sigma \sigma}$ is larger than the exciton-exciton one $T_{\sigma \sigma}^{X}$, despite the presence of the Hopfield factor $X_{\mathbf{k}}^{4}$ in Eq. (16). By comparing Eq. (16) with (17), we see that this enhancement of interactions is purely driven by the difference in scattering energy, which in turn is dominated by the strong light-matter Rabi coupling.

Apart from the enhancement due to the strong light-matter coupling, Fig. 2 shows that the polariton interactions behave qualitatively differently to exciton interactions. At small momenta, the polariton interactions are strongly affected by the Hopfield-factor momentum dependence, and hence the elastic interaction strength initially increases until it reaches a maximum slightly above the inflection wave vector $q_{0}$ of the nonparabolic polariton dispersion. At large wave vectors, the polariton scattering decreases, until it becomes excitonlike and recovers the standard behavior of 2D scattering, as shown in Fig. 2(c).

We emphasize that the peak in the polariton scattering $T$ matrix at finite relative momentum is not due to so-called optical parametric scattering. The present maximum is for scattering at zero total momentum, whereas optical parametric scattering requires a finite total momentum $\sim 2 q_{0}$.

For completeness, in Fig. 2(a) we display the results from the exact expression for polariton-polariton scattering, Eq. (13), as well as the analytic approximation in Eq. (16) 
which relied on the fact that $m_{C} / m_{X} \ll 1$ (dot-dashed black line). We see that these perfectly match for the relevant wave vectors probed in optical experiments, thus proving the validity of our approximations.

\section{B. Polariton interactions at low momentum}

Equation (17) indicates that the exciton interactions vanish logarithmically in the limit of zero momentum. By contrast, this behavior is absent for the polariton interactions shown in Fig. 2. However, in principle the $2 \mathrm{D}$ polariton $T$ matrix must vanish in the limit of strictly zero momentum, as is the case for any scattering of $2 \mathrm{D}$ quantum particles with short-range interactions. As we now explain, this strong qualitative difference between exciton and polariton interactions at very small momentum arises from the large exciton-photon mass ratio, since this implies that the typical momentum at which the polariton interactions start to approach zero is not resolvable in realistic experiments.

We obtained the analytical expression (16) by using the approximation (14), which amounts to replacing the polariton one-loop polarization bubble by the exciton one, as justified in Appendix B. From Eq. (B4) with $E=2 E_{\mathbf{k}}^{L}$ one can see that the leading correction to Eq. (14) at small momentum comes from the first term in the bracket, since this diverges in the zero-momentum limit:

$$
\mathcal{A}=\frac{m_{L}}{4 \pi \hbar^{2}} X_{0}^{4} \ln \left(\frac{2 E_{0}^{L}-E}{-E}\right),
$$

where $m_{L}=m_{C} / C_{0}^{2}$ is the lower polariton effective mass. Keeping this term, the $T$ matrix takes the form

$$
\begin{aligned}
T_{\sigma \sigma^{\prime}}(k \rightarrow 0) & \simeq \frac{X_{\mathbf{k}}^{4}}{\frac{N}{g_{\sigma \sigma^{\prime}}}-N \Pi_{X}\left(2 E_{\mathbf{k}}^{L}\right)-\mathcal{A}} \\
& =\frac{4 \pi \hbar^{2} X_{\mathbf{k}}^{4}}{m_{X} N \ln \left(\frac{\varepsilon_{\sigma \sigma^{\prime}}}{-2 E_{\mathbf{k}}^{L}}\right)-m_{L} \ln \left(\frac{2 E_{0}^{L}-2 E_{\mathbf{k}}^{L}}{-2 E_{\mathbf{k}}^{L}}\right) X_{0}^{4}},
\end{aligned}
$$

and indeed vanishes as

$$
T_{\sigma \sigma^{\prime}} \underset{k \rightarrow 0}{\longrightarrow} \frac{4 \pi \hbar^{2}}{m_{L} \ln \left(\frac{2\left|E_{0}^{L}\right| m_{L}}{-\hbar^{2} k^{2}}\right)} .
$$

One can estimate the wave vector at which this logarithmic behavior starts to dominate by comparing the two terms in the denominator in (19). Using the fact that $\ln \left(\frac{\varepsilon_{\sigma \sigma^{\prime}}}{2\left|E_{0}^{L}\right|}\right) \sim 1$, the second term becomes relevant when

$$
k^{*} a_{\sigma \sigma^{\prime}} \sim \sqrt{\frac{m_{L}}{m_{X}}} e^{-\frac{N m_{X}}{2 m_{L} X_{0}^{4}}} \sim e^{-\frac{N m_{X}}{m_{C}}},
$$

where we have dropped the prefactor and removed the Hopfield coefficients of order 1 in the last term. Because of the large exciton-photon mass ratio, $k^{*}$ is extremely small. For example, for $m_{X} / m_{C}=10^{4}$ and $N=1$, one gets $1 / k^{*} \sim$ $10^{4343} a_{\sigma \sigma^{\prime}}$, corresponding to a length scale much larger than the observable universe radius $\left(\sim 4.4 \times 10^{26} \mathrm{~m}\right)$ ! This explains why the vanishing of $2 \mathrm{D}$ polariton scattering at low momentum is unobservable in any experiment.
It is worth noting that the above estimate strongly differs from the case of binary collisions between ultracold bosonic atoms in quasi-2D geometries [63], for which the logarithmic behavior, albeit challenging to probe experimentally, is not physically impossible to reach.

\section{IMPLICATIONS FOR EXPERIMENTS}

We expect Eq. (1) to be accurate for TMD layers due to the sizable exciton binding energies (see Table I) which imply that $\left|E_{0}^{L}\right| \ll \varepsilon_{B}^{X}$. Figures 3(a)-3(d) show the polariton triplet interaction strength $\alpha_{1}$ for a range of photon-exciton detunings $\delta$ in different 2D TMD systems. Similar to previous predictions within the Born approximation, we find that $\alpha_{1}$ is repulsive and increases with increasing $\delta$ (corresponding to an increasing exciton fraction $X_{0}^{2}$ ). However, we see that a larger number of layers $N$ typically suppresses the polariton triplet interaction strength by a factor of $\sim N$ plus logarithmic corrections, suggesting that the strongest interactions occur in monolayer TMDs, while the largest Rabi coupling is achieved with multiple layers. For the case of monolayer $\mathrm{MoSe}_{2}$, the value of $\alpha_{1}$ at detuning $\delta \simeq-15 \mathrm{meV}$ is consistent with the recent low-density measurement reported in Ref. [28] $\left(\alpha_{1} \simeq\right.$ $0.01 \mu \mathrm{eV} \mu \mathrm{m}^{2}$ ), thus confirming the validity of our model.

In contrast to the triplet case, the polariton singlet interaction strength $\alpha_{2}$ can display a scattering resonance when $2\left|E_{0}^{L}\right| \approx \varepsilon_{+-}$, corresponding to the point where the lower polariton branch crosses the biexciton energy. Such resonances are present in Figs. 3(e)-3(h), where we have plotted the singlet/triplet ratio $\left(\alpha_{2} / \alpha_{1}\right)$ for each TMD system. Here we see that the magnitude and even the sign of $\alpha_{2}$ can be tuned by varying $\delta$ and/or $N$. Furthermore, these panels demonstrate that the singlet interaction is in general stronger than the triplet one for a wide range of experimental parameters. To our knowledge, this important feature has not been noticed previously. In particular, a large and negative $\alpha_{2} / \alpha_{1}$ can destabilize a polariton condensate, which possibly explains why condensation has been challenging to achieve thus far. Based on current experimental data (Table I), our results in Figs. 3(e)-3(h) suggest that $\mathrm{WSe}_{2}$ is the most promising candidate for achieving condensation since it is easiest to access a regime with $\alpha_{2}>0$. Furthermore, the sizable and tunable $\alpha_{2}$ in TMDs opens up the possibility of realizing strongly correlated phenomena such as polariton blockade [64], bipolariton superfluidity [65], and polaron physics [66].

Thus far we have focused our discussion on the case of TMDs, where the large exciton binding energies mean that Eq. (1) is immediately applicable. In conventional quantum well semiconductor microcavities, the ratio of Rabi coupling to exciton binding energy is somewhat larger. For instance, in the case of a single GaAs quantum well, the ratio $\hbar \Omega_{R} / \varepsilon_{B}^{X} \simeq$ 0.35 , and therefore we still expect that our results are reasonably accurate at a quantitative level. Equation (1) then implies that the singlet interaction should be dominant, given the proximity to the biexciton resonance. Indeed this is consistent with experimental results, since the biexciton resonance has only been probed in single GaAs quantum well structures [67-69]. We note that the enhancement of $\alpha_{2}$ in this context, known as the polariton "Feshbach resonance," was proposed in Ref. [70]. On the other hand, for multiple quantum wells, 

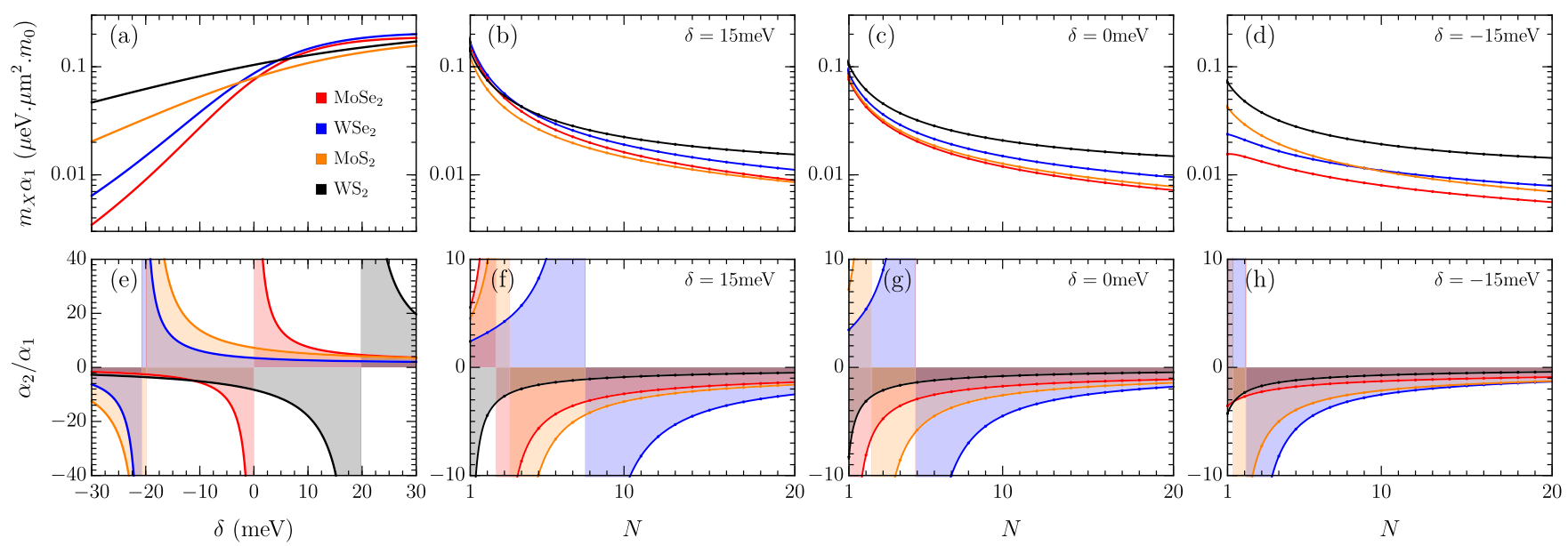

FIG. 3. Polariton interactions in several 2D materials. (a) and (e) Monolayer triplet interactions $\alpha_{1}$ and the corresponding singlet-triplet ratio $\alpha_{2} / \alpha_{1}$ as a function of the exciton-photon detuning $\delta$. (b)-(d) Multilayer triplet interactions as a function of $N$ for different $\delta$, and (f)-(h) the corresponding singlet-triplet ratio. Parameters are taken from Table I, with $m_{0}$ the free electron mass.

such as structures with 12 layers, the Rabi coupling is comparable to the exciton binding energy [56]. While this means that we are formally outside the regime of validity of Eq. (1), we may still draw some qualitative conclusions based on this equation. In particular, from Eq. (1) we see that the enhanced Rabi coupling due to the multiple quantum wells means that the biexciton resonance is off resonant, and therefore the triplet interaction should dominate. This is also consistent with experiment-indeed in experiments with multiple quantum wells the singlet interaction is typically ignored [56].

Note, though, that neither TMDs nor GaAs quantum wells are expected to feature a triplet polariton Feshbach resonance since there is no biexciton in this case: While Eq. (1) naively predicts a resonance at $2\left|E_{0}^{L}\right| \simeq \varepsilon_{B}^{X}$, such a large energy scale goes beyond the validity of our model and requires a more precise description of the short-distance physics such as the composite nature of the excitons and the layer thickness. A full microscopic description of such details is beyond the scope of the present work.

We emphasize that most of the intermediate states appearing in the $T$ matrix are dark states [Fig. 1(b)] which lie at the exciton energy. In our calculation, these correspond to virtual states that only contribute to the final strength of polariton interactions. However, in principle they can be turned into real long-lived excitons via additional scattering processes, for example mediated by phonons [71-73], and they can therefore populate an excitonic reservoir. Furthermore, to our knowledge, spontaneous polariton condensation has never been observed in single-quantum-well microcavities, which suggests that these dark states might also play an important role in the condensation process routinely reported in multiquantum-well structures $[3,4,6]$.

Finally, the finite value of zero-momentum polariton interactions given by Eq. (1) implies that the chemical potential of a polariton condensate will scale linearly with density. This is consistent with recent measurements of the polariton blueshift in the Thomas-Fermi regime [56], and differs from the behavior of a $2 \mathrm{D}$ exciton condensate, which will feature a logarithmic dependence on density according to Bogoliubov theory $[57,74]$. Such a logarithmic dependence has already been observed in condensates of paired fermionic atoms in quasi-2D geometries [75].

\section{SUMMARY AND CONCLUSIONS}

We have derived exact analytical expressions for the polariton-polariton triplet and singlet interaction strengths in TMD layers embedded in a planar microcavity. Crucially, we have demonstrated that the strong exciton-photon coupling enhances the polariton interactions relative to those of bare excitons. The present work, together with the recent demonstration of the enhancement of polariton-electron scattering within a microscopic theory [76,77], suggest that such strengthening of interactions induced by the strong lightmatter coupling is a universal phenomenon in these systems, which has been unnoticed so far.

Furthermore, we have analyzed the dependence on the number of layers and we have exposed the important role of optically dark states in multilayer polariton-polariton scattering. Our results suggest that the singlet interaction is stronger than the triplet one for a range of TMD heterostructures, which has important consequences for realizing polariton condensation and other interaction-driven phenomena in these systems. In particular, a large repulsive singlet interaction can lead to the formation of spin-polarized domains, and thus to spin-resolved ultralow threshold lasing.

Note added in proof. After submission of this manuscript, a related work appeared in which a zero-temperature scalar polariton condensate in a monolayer system was theoretically investigated using a Bogoliubov approach, leading to a similar expression for $\alpha_{1}$ [78].

\section{ACKNOWLEDGMENTS}

We are grateful to F. M. Marchetti, E. Ostrovskaya, and M. Pieczarka for useful discussions. We acknowledge support from the Australian Research Council Centre of Excellence in Future Low-Energy Electronics Technologies (CE170100039). J.L. is also supported through the Australian Research Council Future Fellowship FT160100244. 


\section{APPENDIX A: POLARITON T MATRIX}

Here we provide some details of the $T$-matrix calculation for the scattering between two lower polaritons. First, we recall the bosonic commutation rules:

$$
\left[\hat{A}_{\mathbf{k} \sigma}, \hat{B}_{\mathbf{k}^{\prime} \sigma^{\prime}}^{\dagger}\right]=\delta_{A B} \delta_{\sigma \sigma^{\prime}} \delta_{\mathbf{k k}^{\prime}}
$$

where $\hat{A}, \hat{B}$ can correspond to lower polaritons $\hat{L}$, upper polaritons $\hat{U}$, or dark-exciton operators $\hat{d}_{l}$, with $l=1,2, \ldots, N-1$. The two-particle states with zero total momentum are defined as

$$
\left|A_{\sigma}, B_{\sigma^{\prime}}, \mathbf{k}\right\rangle=\hat{A}_{-\mathbf{k} \sigma}^{\dagger} \hat{B}_{\mathbf{k} \sigma^{\prime}}^{\dagger}|0\rangle
$$

with the scalar product

$$
\begin{aligned}
\left\langle A_{\sigma_{1}}, B_{\sigma_{1}^{\prime}}, \mathbf{k}_{1} \mid C_{\sigma_{2}}, D_{\sigma_{2}^{\prime}}, \mathbf{k}_{2}\right\rangle & =\left\langle 0\left|\hat{B}_{\mathbf{k}_{1} \sigma_{1}^{\prime}} \hat{A}_{-\mathbf{k}_{1} \sigma_{1}} \hat{C}_{-\mathbf{k}_{2} \sigma_{2}}^{\dagger} \hat{D}_{\mathbf{k}_{2} \sigma_{2}^{\prime}}^{\dagger}\right| 0\right\rangle \\
& =\delta_{B C} \delta_{A D} \delta_{\sigma_{1}^{\prime} \sigma_{2}} \delta_{\sigma_{1} \sigma_{2}^{\prime}} \delta_{\mathbf{k}_{1},-\mathbf{k}_{2}}+\delta_{B D} \delta_{A C} \delta_{\sigma_{1} \sigma_{2}} \delta_{\sigma_{1}^{\prime} \sigma_{2}^{\prime}} \delta_{\mathbf{k}_{1} \mathbf{k}_{2}} .
\end{aligned}
$$

The noninteracting eigenvalues are given by

$$
\begin{aligned}
\hat{H}_{0}\left|A_{\sigma}, B_{\sigma^{\prime}}, \mathbf{k}\right\rangle & =\sum_{J} \sum_{\mathbf{q}, s} E_{\mathbf{q}}^{J} \hat{J}_{\mathbf{q} s}^{\dagger} \hat{J}_{\mathbf{q} s} \hat{A}_{-\mathbf{k} \sigma}^{\dagger} \hat{B}_{\mathbf{k} \sigma^{\prime}}^{\dagger}|0\rangle \\
& =\left(E_{-\mathbf{k}}^{A}+E_{\mathbf{k}}^{B}\right) \hat{A}_{-\mathbf{k} \sigma}^{\dagger} \hat{B}_{\mathbf{k} \sigma^{\prime}}^{\dagger}|0\rangle \\
& =\left(E_{-\mathbf{k}}^{A}+E_{\mathbf{k}}^{B}\right)\left|A_{\sigma}, B_{\sigma^{\prime}}, \mathbf{k}\right\rangle
\end{aligned}
$$

where the sum on $J$ in the first line accounts for all the available single-particle energy states, and $E_{\mathbf{q}}^{J} \equiv E_{\mathbf{q}}^{X}$ for all the dark states with $\hat{J}=\hat{d}_{l}$.

Now we consider the scattering between two lower polaritons, the first term of the Born series gives

$$
\left\langle L_{\sigma}, L_{\sigma^{\prime}}, \mathbf{k}^{\prime}|\hat{V}| L_{\sigma}, L_{\sigma^{\prime}}, \mathbf{k}\right\rangle=X_{\mathbf{k}^{\prime}}^{2} X_{\mathbf{k}}^{2} \frac{g_{\sigma \sigma^{\prime}}}{N}\left(1+\delta_{\sigma \sigma^{\prime}}\right) .
$$

(Notice that $g_{+-}=g_{-+}$, and $g_{++}=g_{--}$). Higher order terms in the $T$ matrix involve dark or upper polariton intermediate states. It is useful to introduce the matrix element between two lower polaritons and an arbitrary two-particle state

$$
\left\langle A_{\sigma}, B_{\sigma^{\prime}}, \mathbf{k}^{\prime}|\hat{V}| L_{\sigma}, L_{\sigma^{\prime}}, \mathbf{k}\right\rangle=\frac{g_{\sigma \sigma^{\prime}} X_{\mathbf{k}}^{2}}{N}\left(1+\delta_{\sigma \sigma^{\prime}}\right) \begin{cases}\delta_{\operatorname{Mod}\left[l_{1}+l_{2}, N\right]}, & A=d_{l_{1}}, B=d_{l_{2}}, l_{1}, l_{2} \neq N, \\ X_{\mathbf{k}^{\prime}}^{2}, & A=L, B=L, \\ C_{\mathbf{k}^{\prime}}^{2}, & A=U, B=U, \\ -X_{\mathbf{k}^{\prime}} C_{\mathbf{k}^{\prime}}, & A=L, B=U, \\ -X_{\mathbf{k}^{\prime}} C_{\mathbf{k}^{\prime}}, & A=U, B=L,\end{cases}
$$

and the completeness relation

$$
\mathbb{1}=\frac{1}{2} \sum_{\mathbf{q}} \sum_{A, B} \sum_{s, s^{\prime}}\left|A_{s}, B_{s^{\prime}}, \mathbf{q}\right\rangle\left\langle A_{s}, B_{s^{\prime}}, \mathbf{q}\right|,
$$

which satisfies the usual property of the identity

$$
\begin{aligned}
\mathbb{1}\left|C_{\sigma}, D_{\sigma^{\prime}}, \mathbf{k}\right\rangle & =\frac{1}{2} \sum_{\mathbf{q}} \sum_{A, B} \sum_{s, s^{\prime}}\left|A_{s}, B_{s^{\prime}}, \mathbf{q}\right\rangle\left\langle A_{s}, B_{s^{\prime}}, \mathbf{q} \mid C_{\sigma}, D_{\sigma^{\prime}}, \mathbf{k}\right\rangle \\
& =\frac{1}{2} \sum_{\mathbf{q}}\left(\left|C_{\sigma}, D_{\sigma^{\prime}}, \mathbf{q}\right\rangle \delta_{\mathbf{q k}}+\left|D_{\sigma^{\prime}}, C_{\sigma}, \mathbf{q}\right\rangle \delta_{\mathbf{q},-\mathbf{k}}\right) \\
& =\frac{1}{2}\left(\left|C_{\sigma}, D_{\sigma^{\prime}}, \mathbf{k}\right\rangle+\left|D_{\sigma^{\prime}}, C_{\sigma},-\mathbf{k}\right\rangle\right) \\
& =\left|C_{\sigma}, D_{\sigma^{\prime}}, \mathbf{k}\right\rangle .
\end{aligned}
$$


Then the second-order term reads

$$
\begin{aligned}
\left\langle L^{\sigma}, L^{\sigma^{\prime}}, \mathbf{k}^{\prime}\left|\hat{V} \frac{1}{E-\hat{H}_{0}} \hat{V}\right| L^{\sigma}, L^{\sigma^{\prime}}, \mathbf{k}\right\rangle= & \left\langle L^{\sigma}, L^{\sigma^{\prime}}, \mathbf{k}^{\prime}\left|\hat{V} \mathbb{1} \frac{1}{E-\hat{H}_{0}} \hat{V}\right| L^{\sigma}, L^{\sigma^{\prime}}, \mathbf{k}\right\rangle \\
= & \left(1+\delta_{\sigma \sigma^{\prime}}\right)\left(\frac{g_{\sigma \sigma^{\prime}}}{N}\right)^{2} X_{\mathbf{k}}^{2} X_{\mathbf{k}^{\prime}}^{2} \\
& \times \sum_{\mathbf{q}}\left[\frac{X_{\mathbf{q}}^{4}}{E-2 E_{\mathbf{q}}^{L}}+\frac{C_{\mathbf{q}}^{4}}{E-2 E_{\mathbf{q}}^{U}}+\frac{2 C_{\mathbf{q}}^{2} X_{\mathbf{q}}^{2}}{E-E_{\mathbf{q}}^{L}-E_{\mathbf{q}}^{U}}+\sum_{l_{1}, l_{2}}^{N-1} \frac{\delta_{\operatorname{Mod}\left[l_{1}+l_{2}, N\right]}}{E-2 E_{\mathbf{q}}^{X}}\right] \\
= & \left(1+\delta_{\sigma \sigma^{\prime}}\right) \frac{g_{\sigma \sigma^{\prime}} X_{\mathbf{k}}^{4}}{N} \frac{g_{\sigma \sigma^{\prime}}}{N} \Pi(E),
\end{aligned}
$$

where we have used $\left|\mathbf{k}^{\prime}\right|=|\mathbf{k}|$ in the last line. The one-loop polarization bubble $\Pi(E)$ is given by

$$
\Pi(E)=\underbrace{\sum_{\mathbf{q}}^{\Lambda} \frac{X_{\mathbf{q}}^{4}}{E-2 E_{\mathbf{q}}^{L}}+\sum_{\mathbf{q}}^{\Lambda} \frac{C_{\mathbf{q}}^{4}}{E-2 E_{\mathbf{q}}^{U}}+2 \sum_{\mathbf{q}}^{\Lambda} \frac{X_{\mathbf{q}}^{2} C_{\mathbf{q}}^{2}}{E-E_{\mathbf{q}}^{L}-E_{\mathbf{q}}^{U}}}_{\Pi_{P}(E)}+(N-1) \underbrace{\sum_{\mathbf{q}}^{\Lambda} \frac{1}{E-2 E_{\mathbf{q}}^{X}}}_{\Pi_{X}(E)},
$$

where we have introduced the high-energy cutoff wave vector $\Lambda$. Note that, had we dropped the dark states in Eq. (2) as is commonly done [9], the term $(N-1) \Pi_{X}$ would be absent. Hence the dark states play an essential role in the interactions.

The generalization to higher order terms leads to

$$
\begin{aligned}
\left\langle L_{\sigma}, L_{\sigma^{\prime}}, \mathbf{k}^{\prime}|\hat{T}(E)| L_{\sigma}, L_{\sigma^{\prime}}, \mathbf{k}\right\rangle & =\left(1+\delta_{\sigma \sigma^{\prime}}\right) \frac{g_{\sigma \sigma^{\prime}} X_{\mathbf{k}}^{4}}{N}\left[1+\frac{g_{\sigma \sigma^{\prime}}}{N} \Pi(E)+\left(\frac{g_{\sigma \sigma^{\prime}}}{N} \Pi(E)\right)^{2}+\cdots\right] \\
& =\left(1+\delta_{\sigma \sigma^{\prime}}\right) \frac{X_{\mathbf{k}}^{4}}{\left(\frac{N}{g_{\sigma \sigma^{\prime}}}-\Pi(E)\right)},
\end{aligned}
$$

which after rearranging the prefactor, gives the formula (13) presented in the main text. Note that $\Pi(E)$ also contains a factor $N$. The integrals in $\Pi(E)$ are dominated by the large wave vectors $\mathbf{q}$, where $E_{\mathbf{q}}^{L} \rightarrow E_{\mathbf{q}}^{X}, X_{\mathbf{q}}^{2} \rightarrow 1, C_{\mathbf{q}}^{2} \rightarrow 0$ and one can neglect the second and third terms. Thus, $\Pi(E)$ simply reduces to $N \times \Pi_{X}(E)$. This approximation is valid because $m_{C} \ll m_{X}$ as explained below.

\section{APPENDIX B: $m_{C} \ll m_{X}$ and $\Pi(E)$ APPROXIMATION}

The approximation of the one-loop polarization bubble [Eq. (A18)] relies on the very large difference between the cavity photon and the exciton masses $\left(m_{C} / m_{X} \sim 10^{-4}-10^{-5}\right)$. This approximation is equivalent to neglecting the low- $q$ behavior of the integrand in

$$
\Pi_{P}(E)=\frac{1}{2 \pi} \int_{0}^{\Lambda} q d q\left(\frac{X_{\mathbf{q}}^{4}}{E-2 E_{\mathbf{q}}^{L}}+\frac{C_{\mathbf{q}}^{4}}{E-2 E_{\mathbf{q}}^{U}}+2 \frac{X_{\mathbf{q}}^{2} C_{\mathbf{q}}^{2}}{E-E_{\mathbf{q}}^{L}-E_{\mathbf{q}}^{U}}\right) .
$$

To analytically evaluate if this low-q behavior plays a role, one can approximate the integrand in two domains with the following replacements:

(i) $q<q_{0}: E_{\mathbf{q}}^{L} \rightarrow \tilde{E}_{\mathbf{q}}^{L}=\hbar^{2} q^{2} / 2 m_{L}+E_{0}^{L}, E_{\mathbf{q}}^{U} \rightarrow \tilde{E}_{\mathbf{q}}^{U}=\hbar^{2} q^{2} / 2 m_{U}+E_{0}^{U}, C_{\mathbf{q}} \rightarrow C_{0}, X_{\mathbf{q}} \rightarrow X_{0}$,

(ii) $q>q_{0}: E_{\mathbf{q}}^{L} \rightarrow E_{\mathbf{q}}^{X}, C_{\mathbf{q}} \rightarrow 0, X_{\mathbf{q}} \rightarrow 1$.

The inflection wave vector $q_{0}$ and the lower (upper) polariton effective masses $m_{L},\left(m_{U}\right)$ are defined as

$$
q_{0}=\frac{\sqrt{2 m_{L}\left|E_{0}^{L}\right|}}{\hbar}, \quad m_{L}=\frac{m_{C}}{C_{0}^{2}}, \quad m_{U}=\frac{m_{C}}{X_{0}^{2}} .
$$

This gives

$$
\begin{aligned}
\Pi_{P}(E) \simeq & \frac{1}{2 \pi} \int_{0}^{q_{0}} q d q\left(\frac{X_{0}^{4}}{E-2 \tilde{E}_{\mathbf{q}}^{L}}+\frac{C_{0}^{4}}{E-2 \tilde{E}_{\mathbf{q}}^{U}}+2 \frac{X_{0}^{2} C_{0}^{2}}{E-\tilde{E}_{\mathbf{q}}^{L}-\tilde{E}_{\mathbf{q}}^{U}}\right)+\frac{1}{2 \pi} \int_{q_{0}}^{\Lambda} q d q\left(\frac{1}{E-2 E_{\mathbf{q}}^{X}}\right) \\
= & \frac{m_{C}}{4 \pi \hbar^{2} C_{0}^{2} X_{0}^{2}}\left[X_{0}^{6} \ln \left(\frac{2 E_{0}^{L}-E}{-E}\right)+C_{0}^{6} \ln \left(\frac{2 E_{0}^{U}-E}{2 E_{0}^{U}-E+2\left|E_{0}^{L}\right| X_{0}^{2} / C_{0}^{2}}\right)+4 X_{0}^{4} C_{0}^{4} \ln \left(\frac{(\delta-E) C_{0}^{2} X_{0}^{2}}{C_{0}^{2}(\delta-E)+\left|E_{0}^{L}\right|}\right)\right] \\
& +\frac{m_{X}}{4 \pi \hbar^{2}} \ln \left(\frac{E-2\left|E_{0}^{L}\right| C_{0}^{2} m_{C} / m_{X}}{E-2 E_{\Lambda}^{X}}\right)
\end{aligned}
$$




$$
\simeq \frac{m_{X}}{4 \pi \hbar^{2}} \ln \left(\frac{E}{E-2 E_{\Lambda}^{X}}\right)=\sum_{\mathbf{q}}^{\Lambda} \frac{1}{E-2 E_{\mathbf{q}}^{X}}
$$

Hence, one can see that in the limit $m_{C} / m_{X} \rightarrow 0, \Pi_{P}(E) \rightarrow \Pi_{X}(E)$, and Eq. (A18) reduces to $\Pi(E) \simeq N \Pi_{X}(E)$.

\section{APPENDIX C: TRIPLET EXCITON-EXCITON SCATTERING}

In the main text we assume that the exciton triplet scattering length is of the order of the exciton Bohr radius $a_{B}$. Here we motivate this assumption by recalling that this is the case for $2 \mathrm{D}$ hard disk particles of radius $r_{0}$. For low-energy particles, the two-body scattering is dominated by the $s$ wave contribution, and the center of mass wave function obeys the $2 \mathrm{D}$ radial Schrödinger equation [51]

$$
-\frac{\hbar^{2}}{2 m_{r}} \frac{1}{r} \frac{\partial}{\partial r}\left(r \frac{\partial \psi}{\partial r}\right)+U(r) \psi=E \psi,
$$

with $m_{r}$ the two-body reduced mass. The hard disk potential corresponds to an infinitely high potential barrier of radius $r_{0}$,

$$
U=\left\{\begin{array}{l}
\infty, r \leqslant r_{0} \\
0, \quad r>r_{0}
\end{array}\right.
$$

Outside the potential, the general solution is a superposition of first and second kind Bessel functions

$$
\psi(r)=A J_{0}(k r)+B Y_{0}(k r)
$$

with $k=\sqrt{2 m_{r} E} / \hbar$. Then, using its asymptotic form, one introduces the scattering phase shift $\delta_{s}$,

$$
\psi(r) \underset{r \rightarrow \infty}{\longrightarrow} \sqrt{\frac{2}{\pi k r}}[A \cos (k r-\pi / 4)+B \sin (k r-\pi / 4)]=\sqrt{\frac{2}{\pi k r}} C \cos \left(k r-\pi / 4+\delta_{s}\right),
$$

with

$$
\tan \left(\delta_{s}\right)=-\frac{B}{A}
$$

The continuity of the wave function at $r=r_{0}$ gives the relation

$$
\cot \left(\delta_{s}\right)=-\frac{A}{B}=\frac{Y_{0}\left(k r_{0}\right)}{J_{0}\left(k r_{0}\right)} .
$$

Finally, taking the low-energy (low- $k$ ) limit one obtains

$$
\cot \left(\delta_{s}\right)=\frac{2}{\pi} \ln \left(k a_{s}\right),
$$

where we have introduced the 2D scattering length $a_{s}$,

$$
a_{s}=\frac{e^{\gamma} r_{0}}{2} \simeq 0.89 r_{0}
$$

with $\gamma=0.577 \ldots$ the Euler-Mascheroni constant.

The corresponding hard disk triplet exciton $T$ matrix reads

$$
T_{\sigma \sigma}^{X}(k)=\frac{2 \pi \hbar^{2}}{m_{r}}\left[\ln \left(\frac{\varepsilon_{\sigma \sigma}}{E}\right)+i \pi\right]^{-1},
$$

with

$$
\varepsilon_{\sigma \sigma}=\frac{\hbar^{2}}{2 m_{r} a_{s}^{2}} .
$$

Note that a similar form for the low-energy $T$ matrix has been obtained in Refs. [32,33], both accounting for the composite (electron-hole) nature of the 2D excitons with different theoretical approaches. We emphasize that the exciton-exciton scattering vanishes only at zero momentum [see Figs. 2(b) and 2(c)], and an arbitrary exciton momentum distribution (such as MaxwellBoltzmann) necessarily leads to nonzero scattering. Hence, the above simple formula does not contradict the signatures of exciton-exciton interaction reported in experiments (such as in Ref. [79]). 
[1] P. G. Savvidis, J. J. Baumberg, R. M. Stevenson, M. S. Skolnick, D. M. Whittaker, and J. S. Roberts, Angle-Resonant Stimulated Polariton Amplifier, Phys. Rev. Lett. 84, 1547 (2000).

[2] A. Baas, J. P. Karr, H. Eleuch, and E. Giacobino, Optical bistability in semiconductor microcavities, Phys. Rev. A 69, 023809 (2004).

[3] J. Kasprzak, M. Richard, S. Kundermann, A. Baas, P. Jeambrun, J. M. J. Keeling, F. M. Marchetti, M. H. Szymańska, R. André, J. L. Staehli, V. Savona, P. B. Littlewood, B. Deveaud, and L. S. Dang, Bose-Einstein condensation of exciton polaritons, Nature (London) 443, 409 (2006).

[4] R. Balili, V. Hartwell, D. Snoke, L. Pfeiffer, and K. West, Bose-Einstein condensation of microcavity polaritons in a trap, Science 316, 1007 (2007).

[5] A. Amo, J. Lefrère, S. Pigeon, C. Adrados, C. Ciuti, I. Carusotto, R. Houdré, E. Giacobino, and A. Bramati, Superfluidity of polaritons in semiconductor microcavities, Nat. Phys. 5, 805 (2009).

[6] K. G. Lagoudakis, M. Wouters, M. Richard, A. Baas, I. Carusotto, R. André, L. S. Dang, and B. Deveaud-Plédran, Quantized vortices in an exciton-polariton condensate, Nat. Phys. 4, 706 (2008).

[7] J. Keeling, F. M. Marchetti, M. H. Szymańska, and P. B. Littlewood, Collective coherence in planar semiconductor microcavities, Semicond. Sci. Technol. 22, R1 (2007).

[8] H. Deng, H. Haug, and Y. Yamamoto, Exciton-polariton BoseEinstein condensation, Rev. Mod. Phys. 82, 1489 (2010).

[9] I. Carusotto and C. Ciuti, Quantum fluids of light, Rev. Mod. Phys. 85, 299 (2013).

[10] A. Kavokin, J. J. Baumberg, G. Malpuech, and F. P. Laussy, Microcavities (Oxford University Press, Oxford, 2017).

[11] K. He, N. Kumar, L. Zhao, Z. Wang, K. F. Mak, H. Zhao, and J. Shan, Tightly Bound Excitons in Monolayer $\mathrm{WSe}_{2}$, Phys. Rev. Lett. 113, 026803 (2014).

[12] Z. Ye, T. Cao, K. O'Brien, H. Zhu, X. Yin, Y. Wang, S. G. Louie, and $\mathrm{X}$. Zhang, Probing excitonic dark states in single-layer tungsten disulphide, Nature (London) 513, 214 (2014).

[13] A. Chernikov, T. C. Berkelbach, H. M. Hill, A. Rigosi, Y. Li, O. B. Aslan, D. R. Reichman, M. S. Hybertsen, and T. F. Heinz, Exciton Binding Energy and Nonhydrogenic Rydberg Series in Monolayer $\mathrm{WS}_{2}$, Phys. Rev. Lett. 113, 076802 (2014).

[14] G. Wang, A. Chernikov, M. M. Glazov, T. F. Heinz, X. Marie, T. Amand, and B. Urbaszek, Colloquium: Excitons in atomically thin transition metal dichalcogenides, Rev. Mod. Phys. 90, 021001 (2018).

[15] O. V. Mikhnenko, P. W. M. Blom, and T.-Q. Nguyen, Exciton diffusion in organic semiconductors, Energy Environ. Sci. 8, 1867 (2015).

[16] D. Sanvitto and S. Kéna-Cohen, The road towards polaritonic devices, Nat. Mater. 15, 1061 (2016).

[17] X. Liu, T. Galfsky, Z. Sun, F. Xia, E.-c. Lin, Y.-H. Lee, S. KénaCohen, and V. M. Menon, Strong light-matter coupling in twodimensional atomic crystals, Nat. Photonics 9, 30 (2015).

[18] L. C. Flatten, Z. He, D. M. Coles, A. A. P. Trichet, A. W. Powell, R. A. Taylor, J. H. Warner, and J. M. Smith, Roomtemperature exciton-polaritons with two-dimensional $\mathrm{WS}_{2}$, Sci. Rep. 6, 33134 (2016).

[19] N. Lundt, S. Klembt, E. Cherotchenko, S. Betzold, O. Iff, A. V. Nalitov, M. Klaas, C. P. Dietrich, A. V. Kavokin, S. Höfling, and C. Schneider, Room-temperature Tamm-plasmon exciton- polaritons with a $\mathrm{WSe}_{2}$ monolayer, Nat. Commun. 7, 13328 (2016).

[20] M. Sidler, P. Back, O. Cotlet, A. Srivastava, T. Fink, M. Kroner, E. Demler, and A. Imamoglu, Fermi polaron-polaritons in charge-tunable atomically thin semiconductors, Nat. Phys. 13, 255 (2017).

[21] S. Dufferwiel, S. Schwarz, F. Withers, A. A. P. Trichet, F. Li, M. Sich, O. Del Pozo-Zamudio, C. Clark, A. Nalitov, D. D. Solnyshkov, G. Malpuech, K. S. Novoselov, J. M. Smith, M. S. Skolnick, D. N. Krizhanovskii, and A. I. Tartakovskii, Exciton-polaritons in van der Waals heterostructures embedded in tunable microcavities, Nat. Commun. 6, 8579 (2015).

[22] M. Król, K. Rechcińska, K. Nogajewski, M. Grzeszczyk, K. Łempicka, R. Mirekd, S. Piotrowska, K. Watanabe, T. Taniguchi, M. R. Molas, M. Potemski, J. Szczytko, and B. Pietka, Exciton-polaritons in multilayer $\mathrm{WSe}_{2}$ in a planar microcavity, 2D Mater. 7, 015006 (2019).

[23] C. Schneider, M. M. Glazov, T. Korn, S. Höfling, and B. Urbaszek, Two-dimensional semiconductors in the regime of strong light-matter coupling, Nat. Commun. 9, 2695 (2018).

[24] A. K. Geim and I. V. Grigorieva, van der Waals heterostructures, Nature (London) 499, 419 (2013).

[25] I. A. Shelykh, A. V. Kavokin, Y. G. Rubo, T. C. H. Liew, and G. Malpuech, Polariton polarization-sensitive phenomena in planar semiconductor microcavities, Semicond. Sci. Technol. 25, 013001 (2009).

[26] G. Scuri, Y. Zhou, A. A. High, D. S. Wild, C. Shu, K. De Greve, L. A. Jauregui, T. Taniguchi, K. Watanabe, P. Kim, M. D. Lukin, and H. Park, Large Excitonic Reflectivity of Monolayer $\mathrm{MoSe}_{2}$ Encapsulated in Hexagonal Boron Nitride, Phys. Rev. Lett. 120, 037402 (2018).

[27] F. Barachati, A. Fieramosca, S. Hafezian, J. Gu, B. Chakraborty, D. Ballarini, L. Martinu, V. Menon, D. Sanvitto, and S. Kéna-Cohen, Interacting polariton fluids in a monolayer of tungsten disulfide, Nat. Nanotechnol. 13, 906 (2018).

[28] L. B. Tan, O. Cotlet, A. Bergschneider, R. Schmidt, P. Back, Y. Shimazaki, M. Kroner, and A. İmamoğlu, Interacting PolaronPolaritons, Phys. Rev. X 10, 021011 (2020).

[29] R. P. A. Emmanuele, M. Sich, O. Kyriienko, V. Shahnazaryan, F. Withers, A. Catanzaro, P. M. Walker, F. A. Benimetskiy, M. S. Skolnick, A. I. Tartakovskii, I. A. Shelykh, and D. N. Krizhanovskii, Highly nonlinear trion-polaritons in a monolayer semiconductor, Nat. Commun. 11, 3589 (2020).

[30] J. Gu, V. Walther, L. Waldecker, D. Rhodes, A. Raja, J. C. Hone, T. F. Heinz, S. Kena-Cohen, T. Pohl, and V. M. Menon, Enhanced nonlinear interaction of polaritons via excitonic Rydberg states in monolayer $\mathrm{WSe}_{2}$, arXiv:1912.12544.

[31] M. Waldherr, N. Lundt, M. Klaas, S. Betzold, M. Wurdack, V. Baumann, E. Estrecho, A. Nalitov, E. Cherotchenko, H. Cai, E. A. Ostrovskaya, A. V. Kavokin, S. Tongay, S. Klembt, S. Höfling, and C. Schneider, Observation of bosonic condensation in a hybrid monolayer $\mathrm{MoSe}_{2}-\mathrm{GaAs}$ microcavity, Nat. Commun. 9, 3286 (2018).

[32] R. Takayama, N. H. Kwong, I. Rumyantsev, M. KuwataGonokami, and R. Binder, T-matrix analysis of biexcitonic correlations in the nonlinear optical response of semiconductor quantum wells, European Phys. J. B 25, 445 (2002).

[33] C. Schindler and R. Zimmermann, Analysis of the excitonexciton interaction in semiconductor quantum wells, Phys. Rev. B 78, 045313 (2008). 
[34] S. K. Adhikari, Quantum scattering in two dimensions, Am. J. Phys. 54, 362 (1986).

[35] J. Levinsen and M. M. Parish, Strongly interacting twodimensional Fermi gases, Annu. Rev. Cold Atoms Mol. 3, 1 (2015).

[36] C. Ciuti, V. Savona, C. Piermarocchi, A. Quattropani, and P. Schwendimann, Role of the exchange of carriers in elastic exciton-exciton scattering in quantum wells, Phys. Rev. B 58, 7926 (1998).

[37] F. Tassone and Y. Yamamoto, Exciton-exciton scattering dynamics in a semiconductor microcavity and stimulated scattering into polaritons, Phys. Rev. B 59, 10830 (1999).

[38] M. Z. Maialle, E. A. de Andrada e Silva, and L. J. Sham, Exciton spin dynamics in quantum wells, Phys. Rev. B 47, 15776 (1993).

[39] M. M. Glazov, T. Amand, X. Marie, D. Lagarde, L. Bouet, and B. Urbaszek, Exciton fine structure and spin decoherence in monolayers of transition metal dichalcogenides, Phys. Rev. B 89, 201302(R) (2014).

[40] T. Yu and M. W. Wu, Valley depolarization due to intervalley and intravalley electron-hole exchange interactions in monolayer $\mathrm{MoS}_{2}$, Phys. Rev. B 89, 205303 (2014).

[41] G. Panzarini, L. C. Andreani, A. Armitage, D. Baxter, M. S. Skolnick, V. N. Astratov, J. S. Roberts, A. V. Kavokin, M. R. Vladimirova, and M. A. Kaliteevski, Exciton-light coupling in single and coupled semiconductor microcavities: Polariton dispersion and polarization splitting, Phys. Rev. B 59, 5082 (1999).

[42] A. Kavokin, G. Malpuech, and M. Glazov, Optical Spin Hall Effect, Phys. Rev. Lett. 95, 136601 (2005).

[43] C. Leyder, M. Romanelli, J. P. Karr, E. Giacobino, T. C. H. Liew, M. M. Glazov, A. V. Kavokin, G. Malpuech, and A. Bramati, Observation of the optical spin Hall effect, Nat. Phys. 3, 628 (2007).

[44] O. Bleu, D. D. Solnyshkov, and G. Malpuech, Optical valley Hall effect based on transitional metal dichalcogenide cavity polaritons, Phys. Rev. B 96, 165432 (2017).

[45] N. Lundt, Ł. Dusanowski, E. Sedov, P. Stepanov, M. M. Glazov, S. Klembt, M. Klaas, J. Beierlein, Y. Qin, S. Tongay, M. Richard, A. V. Kavokin, S. Höfling, and C. Schneider, Optical valley Hall effect for highly valley-coherent exciton-polaritons in an atomically thin semiconductor, Nat. Nanotechnol. 14, 770 (2019).

[46] E. Ivchenko, A. Nesvizhskii, and S. Jorda, Resonant Bragg reflection from quantum-well structures, Superlattices Microstruct. 16, 17 (1994).

[47] A. Kavokin and G. Malpuech, Cavity Polaritons (Elsevier, Amsterdam, 2003), Vol. 32.

[48] A similar transformation has been considered in Ref. [80], but its explicit mathematical form was not given.

[49] X.-X. Zhang, Y. You, S. Y. F. Zhao, and T. F. Heinz, Experimental Evidence for Dark Excitons in Monolayer $\mathrm{WSe}_{2}$, Phys. Rev. Lett. 115, 257403 (2015).

[50] G. Wang, C. Robert, M. M. Glazov, F. Cadiz, E. Courtade, T. Amand, D. Lagarde, T. Taniguchi, K. Watanabe, B. Urbaszek, and X. Marie, In-Plane Propagation of Light in Transition Metal Dichalcogenide Monolayers: Optical Selection Rules, Phys. Rev. Lett. 119, 047401 (2017).

[51] L. D. Landau and E. M. Lifshitz, Quantum Mechanics: Nonrelativistic Theory (Elsevier, Amsterdam, 2013), Vol. 3.
[52] G. Rochat, C. Ciuti, V. Savona, C. Piermarocchi, A. Quattropani, and P. Schwendimann, Excitonic Bloch equations for a two-dimensional system of interacting excitons, Phys. Rev. B 61, 13856 (2000).

[53] M. Combescot, O. Betbeder-Matibet, and F. Dubin, The manybody physics of composite bosons, Phys. Rep. 463, 215 (2008).

[54] M. M. Glazov, H. Ouerdane, L. Pilozzi, G. Malpuech, A. V. Kavokin, and A. D'Andrea, Polariton-polariton scattering in microcavities: A microscopic theory, Phys. Rev. B 80, 155306 (2009).

[55] V. Shahnazaryan, I. Iorsh, I. A. Shelykh, and O. Kyriienko, Exciton-exciton interaction in transition-metal dichalcogenide monolayers, Phys. Rev. B 96, 115409 (2017).

[56] E. Estrecho, T. Gao, N. Bobrovska, D. Comber-Todd, M. D. Fraser, M. Steger, K. West, L. N. Pfeiffer, J. Levinsen, M. M. Parish, T. C. H. Liew, M. Matuszewski, D. W. Snoke, A. G. Truscott, and E. A. Ostrovskaya, Direct measurement of polariton-polariton interaction strength in the Thomas-Fermi regime of exciton-polariton condensation, Phys. Rev. B 100, 035306 (2019).

[57] C. Mora and Y. Castin, Ground State Energy of the TwoDimensional Weakly Interacting Bose Gas: First Correction Beyond Bogoliubov Theory, Phys. Rev. Lett. 102, 180404 (2009).

[58] I. Kylänpää and H.-P. Komsa, Binding energies of exciton complexes in transition metal dichalcogenide monolayers and effect of dielectric environment, Phys. Rev. B 92, 205418 (2015).

[59] Y. You, X.-X. Zhang, T. C. Berkelbach, M. S. Hybertsen, D. R. Reichman, and T. F. Heinz, Observation of biexcitons in monolayer $\mathrm{WSe}_{2}$, Nat. Phys. 11, 477 (2015).

[60] K. Hao, J. F. Specht, P. Nagler, L. Xu, K. Tran, A. Singh, C. K. Dass, C. Schüller, T. Korn, M. Richter et al., Neutral and charged inter-valley biexcitons in monolayer $\mathrm{MoSe}_{2}$, Nat. Commun. 8, 15552 (2017).

[61] C. Mai, A. Barrette, Y. Yu, Y. G. Semenov, K. W. Kim, L. Cao, and K. Gundogdu, Many-body effects in valleytronics: Direct measurement of valley lifetimes in single-layer $\mathrm{MoS}_{2}$, Nano Lett. 14, 202 (2014).

[62] P. Nagler, M. V. Ballottin, A. A. Mitioglu, M. V. Durnev, T. Taniguchi, K. Watanabe, A. Chernikov, C. Schüller, M. M. Glazov, P. C. M. Christianen, and T. Korn, Zeeman Splitting and Inverted Polarization of Biexciton Emission in Monolayer $\mathrm{WS}_{2}$, Phys. Rev. Lett. 121, 057402 (2018).

[63] D. S. Petrov and G. V. Shlyapnikov, Interatomic collisions in a tightly confined Bose gas, Phys. Rev. A 64, 012706 (2001).

[64] I. Carusotto, T. Volz, and A. Imamoğlu, Feshbach blockade: Single-photon nonlinear optics using resonantly enhanced cavity polariton scattering from biexciton states, Europhys. Lett. 90, 37001 (2010).

[65] F. M. Marchetti and J. Keeling, Collective Pairing of Resonantly Coupled Microcavity Polaritons, Phys. Rev. Lett. 113, 216405 (2014).

[66] J. Levinsen, F. M. Marchetti, J. Keeling, and M. M. Parish, Spectroscopic Signatures of Quantum Many-Body Correlations in Polariton Microcavities, Phys. Rev. Lett. 123, 266401 (2019).

[67] N. Takemura, S. Trebaol, M. Wouters, M. T. Portella-Oberli, and B. Deveaud, Polaritonic Feshbach resonance, Nat. Phys. 10, 500 (2014).

[68] M. Vladimirova, S. Cronenberger, D. Scalbert, K. V. Kavokin, A. Miard, A. Lemaître, J. Bloch, D. Solnyshkov, G. Malpuech, 
and A. V. Kavokin, Polariton-polariton interaction constants in microcavities, Phys. Rev. B 82, 075301 (2010).

[69] N. Takemura, M. D. Anderson, M. Navadeh-Toupchi, D. Y. Oberli, M. T. Portella-Oberli, and B. Deveaud, Spin anisotropic interactions of lower polaritons in the vicinity of polaritonic Feshbach resonance, Phys. Rev. B 95, 205303 (2017).

[70] M. Wouters, Resonant polariton-polariton scattering in semiconductor microcavities, Phys. Rev. B 76, 045319 (2007).

[71] C. Piermarocchi, F. Tassone, V. Savona, A. Quattropani, and P. Schwendimann, Nonequilibrium dynamics of free quantumwell excitons in time-resolved photoluminescence, Phys. Rev. B 53, 15834 (1996).

[72] I. G. Savenko, T. C. H. Liew, and I. A. Shelykh, Stochastic Gross-Pitaevskii Equation for the Dynamical Thermalization of Bose-Einstein Condensates, Phys. Rev. Lett. 110, 127402 (2013).

[73] P. Stepanov, I. Amelio, J.-G. Rousset, J. Bloch, A. Lemaître, A. Amo, A. Minguzzi, I. Carusotto, and M. Richard, Dispersion relation of the collective excitations in a resonantly driven polariton fluid, Nat. Commun. 10, 3869 (2019).

[74] V. N. Popov, On the theory of the superfluidity of two- and onedimensional Bose systems, Theor. Math. Phys. 11, 565 (1972).
[75] I. Boettcher, L. Bayha, D. Kedar, P. A. Murthy, M. Neidig, M. G. Ries, A. N. Wenz, G. Zürn, S. Jochim, and T. Enss, Equation of State of Ultracold Fermions in the 2D BEC-BCS Crossover Region, Phys. Rev. Lett. 116, 045303 (2016).

[76] G. Li, O. Bleu, M. M. Parish, and J. Levinsen, Enhanced scattering between electrons and exciton-polaritons in a microcavity arXiv:2008.09281.

[77] G. Li, J. Levinsen, and M. M. Parish, Theory of polariton-electron interactions in semiconductor microcavities arXiv:2008.09292.

[78] H. Hu, H. Deng, and X.-J. Liu, Two-dimensional exciton-polariton interactions beyond the Born approximation arXiv:2004.05559 (2020).

[79] G. Moody, C. Kavir Dass, K. Hao, C.-H. Chen, L.-J. Li, A. Singh, K. Tran, G. Clark, X. Xu, G. Berghäuser, E. Malic, A. Knorr, and X. Li, Intrinsic homogeneous linewidth and broadening mechanisms of excitons in monolayer transition metal dichalcogenides, Nat. Commun. 6, 8315 (2015).

[80] G. C. L. Rocca, F. Bassani, and V. M. Agranovich, Biexcitons and dark states in semiconductor microcavities, J. Opt. Soc. Am. B 15, 652 (1998). 